\title{
Plasmon-enhanced four-wave mixing by nanoholes in thin gold films
}

\author{
Henning Hagman, ${ }^{1}$ Olof Bäcke, ${ }^{1}$ Juris Kiskis, ${ }^{1}$ Fredrik Svedberg, ${ }^{1}$ Magnus P. Jonsson, ${ }^{2}$ \\ Fredrik Höök, ${ }^{3}$ and Annika Enejder ${ }^{1, *}$ \\ ${ }^{1}$ Department of Chemical and Biological Engineering, Chalmers University of Technology, SE-412 96 Gothenburg, Sweden \\ ${ }^{2}$ Kavli Institute of Nanoscience, Delft University of Technology, Lorentzweg 1, 2628 CJ Delft, The Netherlands \\ ${ }^{3}$ Department of Applied Physics, Chalmers University of Technology, SE-412 96 Gothenburg, Sweden \\ ${ }^{*}$ Corresponding author: enejder@chalmers.se
}

Received September 4, 2013; revised December 17, 2013; accepted January 12, 2014; posted January 14, 2014 (Doc. ID 196959); published February 12, 2014

\begin{abstract}
Nonlinear plasmonics opens up for wavelength conversion, reduced interaction/emission volumes, and nonlinear enhancement effects at the nanoscale with many compelling nanophotonic applications foreseen. We investigate nonlinear plasmonic responses of nanoholes in thin gold films by exciting the holes individually with tightly focused laser beams, employing a degenerated pump/probe and Stokes excitation scheme. Excitation of the holes results in efficient generation of both narrowband four-wave mixing (FWM) and broadband multiphoton excited luminescence, blueshifted relative to the excitation beams. Clear enhancements were observed when matching the pump/probe wavelength with the hole plasmon resonance. These observations show that the FWM generation is locally excited by nanoholes and has a resonant behavior primarily governed by the dimensions of the individual holes. (C) 2014 Optical Society of America

OCIS codes: (250.5403) Plasmonics; (310.6628) Subwavelength structures, nanostructures; (180.4315) Nonlinear microscopy; (300.2570) Four-wave mixing.

http://dx.doi.org/10.1364/OL.39.001001
\end{abstract}

Nanostructures provide a unique and effective means to concentrate and manipulate light at the nanoscale through the excitation of collective electron oscillations known as surface plasmons. While so-called surface plasmon polaritons (SPPs) propagate along metal surfaces, metal nanostructures can also sustain spatially confined localized surface plasmons (LSPs), the resonances (LSPRs) of which depend on the size, shape and, higher-order arrangements of the nanostructures. While the linear regime of plasmonics has been extensively investigated over the past decade, the nonlinear, multiphoton excited correspondent remains relatively unexplored. The idea of downscaling nonlinear interaction phenomena by inducing nonlinear plasmonic effects in nanosized objects is highly attractive, as they could in contrast to the linear correspondents, generate coherent fields, offer frequency conversion and nonlinear enhancement effects, presenting new dimensions in the development of laser-like nanoemitters, nanosized devices for long-range information transfer/optical storage, and nanosensors [믈 $\underline{6}$. The experimentally most straightforward schemes of nonlinear plasmon excitation, second- and higher-harmonic generation as well as multiphoton excited luminescence (MPEL), have been studied on different nanostructures [7-13]. More recently, a series of reports on the more complex but interesting four-wave mixing (FWM) process has appeared [14-19].

In one of the most common FWM excitation schemes, two of the three initial fields are degenerate, requiring only two excitation beams: the pump/probe and Stokes at wavelengths $\lambda_{1}$ and $\lambda_{2}$, respectively (where $\lambda_{1}<\lambda_{2}$ ). This results in a blueshifted FWM signal emitted at $1 / \lambda_{\text {FWM }}=2 / \lambda_{1}-1 / \lambda_{2} \quad[$ Fig. $\underline{1(a)}]$. Furthermore, the FWM intensity, $I_{\mathrm{FWM}}$, depends quadratically on the intensity of the pump/probe beam, $I_{1}$, and linearly on the intensity of the Stokes beam, $I_{2}: I_{\mathrm{FWM}} \propto I_{1}^{2} \times I_{2}$, resulting in strong signal generation though spatially localized to high excitation intensity regions only. In smooth gold films excitation of SPPs at FWM frequency have been shown for free-space excitation [15], requiring fine tuning of the angle between the excitation beams and the angle of the beams relative to the film, and by collinear beams in Kretschmann configuration $[14,16]$. In contrast to excitation of SPPs in plane films, requiring momentum matching, nanostructures also sustain nonpropagating LSPs, requiring wavelength matching to the LSPR. Plasmonenhanced FWM with focused excitation beams has been shown for highly ordered nanostructures, such as gold zig-zag nanowires [17] and structured gold surfaces $[\underline{18,19] .}$.

In linear plasmonics, nanoholes in thin films are well investigated, and promising substrates for biosensing applications as their LSPRs have been shown to display very competitive sensitivity to changes of index of refraction $[20,21]$. The practical applicability of short-range order hole arrays also benefits from the fact that they can be both easily and cost efficiently fabricated with varying hole diameter and density over large areas [5]. In light of their emergent use in sensing application, fundamental investigations of their optical properties are important.

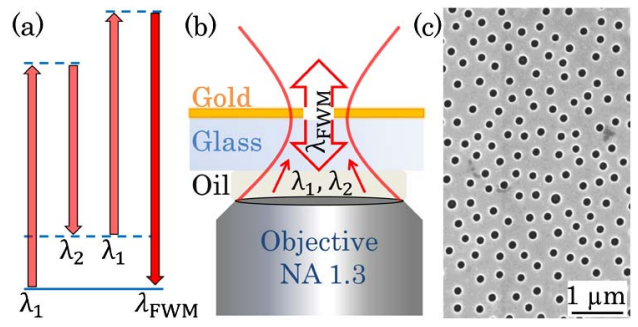

Fig. 1. (a) Energy level diagram of the FWM process. (b) Schematic of the excitation geometry. (c) SEM image of a gold film with a high density of holes; diameter here $140 \mathrm{~nm}$. 
Here, studies of FWM in such substrate could offer insight with regards to frequency conversion efficiency and dependence on plasmonic resonances. Such investigations may also in their own right lead to applications not yet realized. Moreover, by matching the frequency difference of the two excitation beams with a resonant molecular vibration, enhanced emission can be achieved (coherent anti-Stokes Raman scattering, CARS), enabling specific and sensitive vibrational detection [22]. A good understanding of the plasmonic resonances for nonlinear processes in nanoholes is therefore also of great interest for future plasmonic sensors including surface-enhanced CARS emission.

In the current work, we use the tight-focusing excitation scheme to study nonlinear enhancements by shortrange ordered nanoholes in a gold film, illustrating their potential as broadband (MPEL) and coherent narrowband (FWM) nanoemitters, as well as highly localized nanoscale sensors. The small focal volume allows probing individual nanostructures close by each other. We examine whether their nonlinear response is sufficient to generate a detectable signal at excitation powers that the gold film tolerates, both as well separated single units and as a dense population. By varying the excitation wavelengths for five different hole diameters, resonant phenomena and their origin are investigated and discussed in terms of their use as small-scale sensor elements and laser-like nanoemitters.

Thin gold films with nanoholes were manufactured using a convenient colloidal lithography technique [23]. A $1 \mathrm{~nm}$ thick layer of $\mathrm{Cr}$ was used to ensure good adhesion between the glass and the gold. Two sets of films were manufactured with low and high hole densities, respectively. The low density films, $50 \mathrm{~nm}$ thick, with a distance of $5-10 \mu \mathrm{m}$ between the $150 \mathrm{~nm}$ diameter holes, were used to address and investigate the efficacy of single holes. The high hole density films were $30 \mathrm{~nm}$ thick, with hole diameters of $80,110,140,170$, and $190 \mathrm{~nm}( \pm 5 \%)$. The average area coverage was kept constant at roughly $12 \%$, leading to an average distance to closest neighbor of $170 \mathrm{~nm}$ for the $80 \mathrm{~nm}$ hole-diameter film, and up to $380 \mathrm{~nm}$ for the $190 \mathrm{~nm}$ hole-diameter film. An electron microscopy image of the $140 \mathrm{~nm}$ diameter hole film is shown in Fig. 1(c).

The experiments were conducted using a conventional nonlinear microscope, described in detail elsewhere [24]. Briefly, laser beams from a pump laser (1064 nm, Nd: Vanadate, $7 \mathrm{ps}, 76 \mathrm{MHz}$, Picotrain, HighQ Lasers) and an optical parametric oscillator (700-980 nm OPO; Emerald, APE) were spatially and temporally overlapped and guided into an inverted laser scanning microscope (Eclipse TE-2000-E, Nikon). The $1064 \mathrm{~nm}$ output was used as the Stokes beam $\left(\lambda_{2}\right)$, resulting in a FWM signal at 562,663 , and $755 \mathrm{~nm}$ when being overlapped with the $\lambda_{1}$ beam from the OPO at 735,817 , and $883 \mathrm{~nm}$, respectively. The OPO beam was also used to induce MPEL. Excitation powers were approximately $2 \mathrm{~mW}$ for $\lambda_{1}$ and $4 \mathrm{~mW}$ for $\lambda_{2}$ at the sample. The beams were focused onto the sample by a high NA objective (Plan Fluor $\times 40,1.3$ NA, Nikon), resulting in a large distribution of excitation angles [Fig. 1(b)] relaxing FWM phase-matching requirements. The high-NA objective formed a small diffraction-limited focal volume, allowing highly localized excitation. Laser beams were raster scanned over the sample and the nonlinear emission was collected either in the forward direction by a condenser lens, or in the epi direction by the objective. Since detection is not confocal, the detected signal is collected from a relatively large area $(\sim 150 \mu \mathrm{m}$ diameter $)$, although the excitation is strongly local. Therefore, the images primarily give information about where excitation occurs. The nonlinear signals were filtered out by suitable optical bandpass filters and recorded by single photon counting photomultiplier tubes (PMC-100-20, Becker \& Hickl). Extinction and emission spectra recorded from high hole density films were obtained in transmission and epi modes, respectively, using a spectrometer (BRC711E, B\&WTek). For the extinction spectra, a tungsten-halogen lamp (HL2000, Ocean Optics) was used.

As a first investigation of the nonlinear emission from nanoholes, spectra were collected in the epi direction while exciting with the combination of 817 and $1064 \mathrm{~nm}$ in laser scan mode. A spectrum of the sample (hole diameter $140 \mathrm{~nm}$ ) shown in Fig. 1(c) is exemplified in Fig. 2(e). It reveals a strong and narrow component at the FWM wavelength $(663 \mathrm{~nm})$ on top of a broadband component representing the MPEL. In addition, a small peak at $532 \mathrm{~nm}$ can be noted, indicating that the nanoholes also couple out second-harmonic generation (SHG) induced by the $1064 \mathrm{~nm}$ excitation beam. SHG induced by the $817 \mathrm{~nm}$ excitation beam falls outside the detected wavelength region. After compensation for limited spectral resolution of the spectrometer, the spectral intensity at the FWM wavelength $(663 \mathrm{~nm})$ is a factor of $2 \times 10^{2}$ higher for FWM compared to MPEL. A quadratic dependence of the FWM signal on the pump/probe power, and linear for Stokes, is confirmed [insert Fig. 2(e)].

To investigate local response of individual nanoholes, a low hole density film was scanned using the combination of 817 and $1064 \mathrm{~nm}$ beams. FWM emission (bandpass filter $663 \pm 20 \mathrm{~nm}$ ) from single nanoholes could clearly be distinguished, both in epi and forward directions [Figs. 2(a) and 2(b)]. By comparing the intensity of the FWM signal from the nanoholes and the film, we conclude that the holes generate approximately a factor of $10^{2}$ higher signals.

While the spatial positions of the emitted FWM signal in the epi direction [Fig. 2(a)] and in the forward direction [Fig. 2(b)] overlap precisely, the detected signal is significantly lower in the forward direction, $I_{\text {epi }} / I_{\text {forward }} \approx 4$ [Fig. 2(f)]. The higher signal in the epi direction could be explained by more efficient out-coupling due to the asymmetric dielectric environment of the thin gold film (glass-gold-air), together with different collection geometries (the high-NA objective for epi collection compared to the open air condenser in the forward direction). Similar asymmetry was also observed for the MPEL [Fig. 2(f)]. The MPEL image [Fig. 2(c), same region as in Figs. 2(a) and 2(b)] was collected by exciting with the $817 \mathrm{~nm}$ beam only. The nanoholes are still visible at the same positions as for the FWM signal though at a 5 times lower signal [integrated over a wavelength band of $\pm 20 \mathrm{~nm}$ around $663 \mathrm{~nm}$, confirming the factor 200 per spectral unit as deduced from the spectrum in Fig. 2(e)]. Forward detection of MPEL shows the same structures, but yet another factor of 4 weaker (data not shown). 

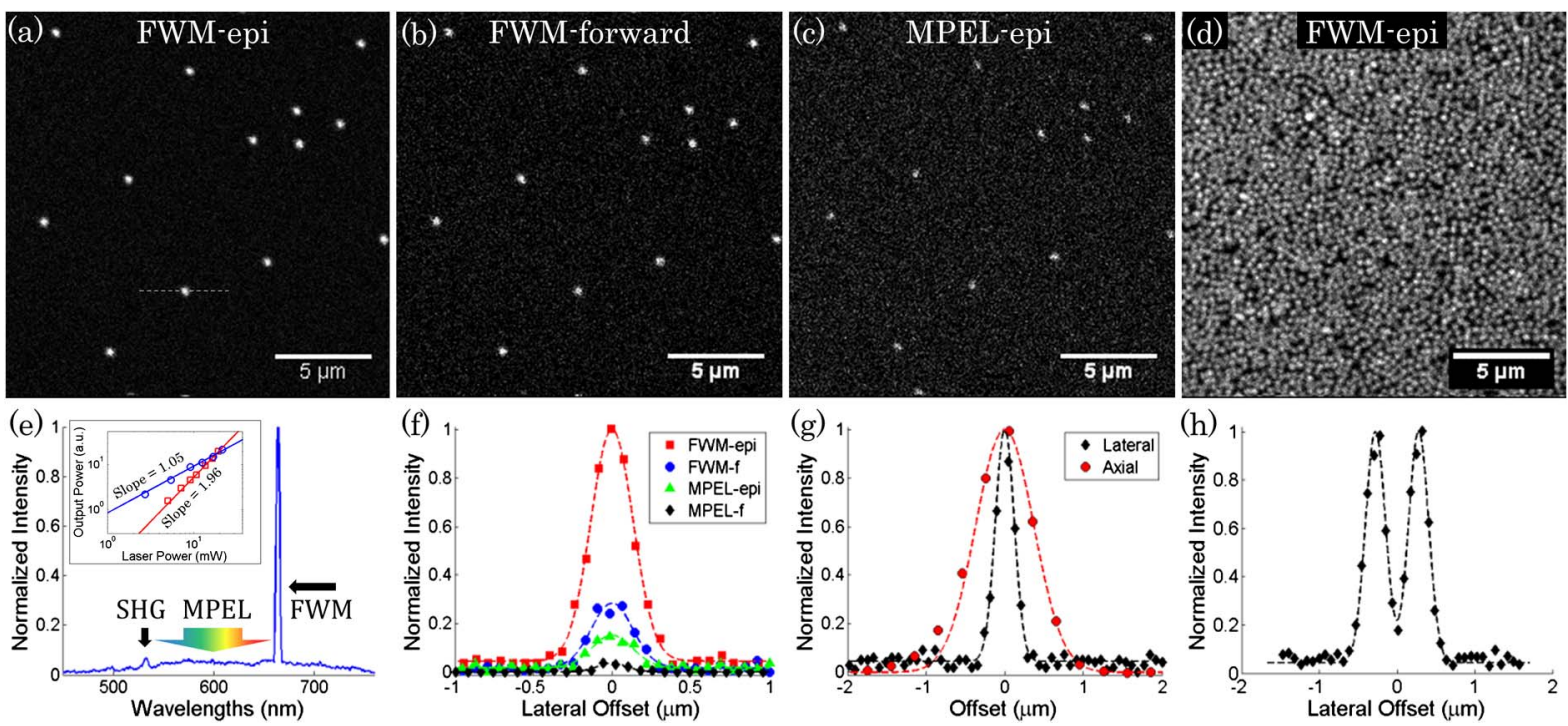

Fig. 2. Images of thin gold films with nanoholes $\left(\lambda_{1}=817 \mathrm{~nm}, \lambda_{2}=1064 \mathrm{~nm}, \lambda_{\mathrm{FWM}}=663 \mathrm{~nm}\right.$, detection at $\left.663 \pm 20 \mathrm{~nm}\right)$. FWM signal collected from the low hole-density film (D: $150 \mathrm{~nm}$ ) in (a) epi and (b) forward. (c) Corresponding MPEL image (epi, excitation only $\lambda_{1}$ ). (d) FWM image of the high-density film shown in Fig. 1 (epi). (e) Spectrum from the high-density gold film in (d). Inset: Output powers versus input powers on log-log scale (blue: Stokes beam, slope $=1.05$, red: pump/probe, slope $=1.96$ ). (f) Intensity profile plots over a single hole [dashed line in (a)]. (g) Lateral and axial profiles of a single hole, indicating the corresponding resolutions (0.3 and $0.9 \mu \mathrm{m})$. (h) Lateral profile for two adjacent holes. In (f)-(h), dashed lines are Gaussian fits.

Upon excitation with the Stokes beam at $1064 \mathrm{~nm}$ only, the emission of MPEL from the $150 \mathrm{~nm}$ diameter holes is insignificant and no holes are visible (data not shown).

To investigate how local the excitation is, beams were scanned along a line over a single hole in both the lateral and axial directions [Fig. 2(g)]. The FWHM of the generated signal is found to be approximately $300 \mathrm{~nm}$ in the lateral plane and $\sim 900 \mathrm{~nm}$ along the axial. These results indicate that efficient FWM excitation occurs only when excitation beams are centered over the hole. In Fig. 2(h) a lateral intensity profile plot over two adjacent holes separated by merely $570 \mathrm{~nm}$ is shown. They can clearly be resolved and fitted by two Gaussian curves, indicating that the excitation is local rather than involving neighboring holes. This conclusion is strengthened by the FMW image of the high-density film [Fig. 2(d)] where the single holes clearly can be resolved despite their short holehole distance. Altogether this means that dense collections of nanoholes can be excited as separate units with tightly focused laser beams without any visible coupling effects between the holes, opening up for dense arrays of individual nanoemitters and nanosensors.

To investigate the FWM/MPEL dependence on the plasmonic resonances of the holes, extinction spectra (wide-field transmission, probing multiple holes simultaneously) of a series of films with different hole diameters were acquired [Fig. 3(a)]. These resonances are well described in the literature for the linear regime [6]. The extinction peaks can be associated with Bloch wave SPPs (BW-SPP), the resonances of which depend on the holehole distance, while the subsequent dips in the extinction can be associated with the hole plasmon resonance (HPR) at a wavelength $\left(\lambda_{\text {HPR }}\right)$ dependent on the hole geometry. Due to the small focal volume of tightly focused excitation beams the HPR is assumed to be more important than the BW-SPPs for the excitation of plasmons. In the case of circular hole, optimal coupling between light polarized parallel to the surface occurs for film plasmons of half-wavelength equal to the diameter of the hole. The central wavelength of the HPR (dips) is expected to have approximately linear dependence on the hole-diameter/film-thickness ratio [25,26], confirmed by the linear fit in Fig. 3(c) showing the hole diameter versus the dip wavelengths $\left(\lambda_{\mathrm{HPR}}\right)$ extracted from Fig 3(a).

In Fig. 3(b) the average FWM count rates for five hole diameters and three $\lambda_{1}(735,817$, and $883 \mathrm{~nm})$ are compared under the same experimental conditions. Error bars describe variations between holes (spread in hole geometry, laser fluctuations, shot noise). Each hole diameter is shown to have an optimal wavelength set $\left(\lambda_{1}, \lambda_{2}, \lambda_{\mathrm{FWM}}\right)$, which are plotted in Fig. 3(d), together with $\lambda_{\text {HPR }}$ deduced in Fig. 3(c), showing good overlap with the $\lambda_{1}$. A similar behavior is also evident for MPEL (excited by only $\lambda_{1}$ ) where the emission intensities peak at the same hole diameters as for the corresponding FWM [Figs. 3(e) and 3(f)]. A strong, and highly similar, dependence of FWM and MPEL intensities on the matching of $\lambda_{1}$ to the plasmonic resonance could be expected due to the emission intensities nonlinear (quadratic) power dependence on this wavelength, and are in agreement with earlier FWM studies [17-19]. The good correlation between $\lambda_{1}$ and $\lambda_{\mathrm{HPR}}$ strengthens the assumption that for the plasmon excitation the HPR is more important than the BW-SPPs, and that the excitation hence is highly local and primarily addresses single holes. Our study does not give spatial information about the emission. If emission from many holes simultaneously occurs, also the BWSPP could affect the FWM conversion efficiency. Further studies with better spatial information of the emission could be achieved by imaging the surface using a CCD camera, complemented by near-field scanning optical microscopy (NSOM). NSOM experiments could also provide important information on the optical near-field 

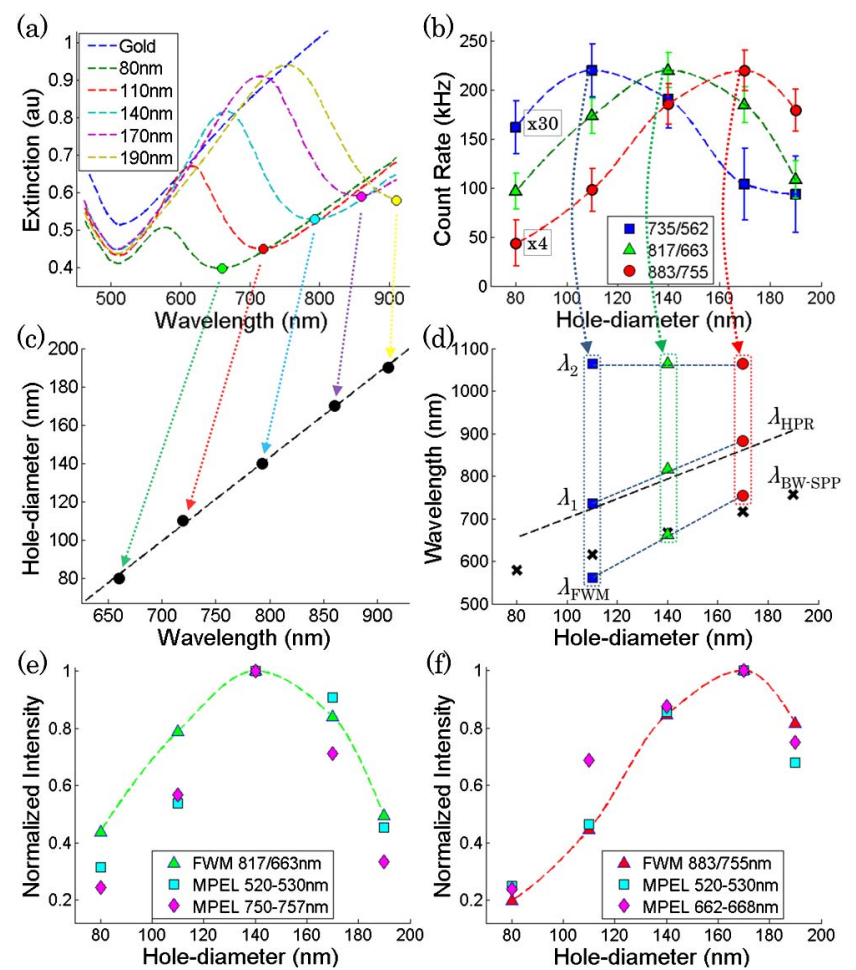

Fig. 3. (a) Extinction spectra for the five hole diameters. Circles indicate the extinction minimum $\left(\lambda_{\mathrm{HPR}}\right)$. (b) Normalized FWM intensities versus hole diameter for three $\lambda_{1}$ (legend: $\lambda_{1} / \lambda_{\text {FWM }}$ ). (c) Relation between $\lambda_{\mathrm{HPR}}$ and hole diameter. (d) The sets of $\left(\lambda_{1}, \lambda_{2}\right.$, and $\left.\lambda_{\text {FWM }}\right)$ are plotted at the hole diameter where highest FWM conversion efficiency was obtained. The center wavelength of the HPR and the BW-SPPs are shown as a dashed line and as crosses, respectively. (e) and (f) MPEL emission versus hole diameter, excited by 817 and $883 \mathrm{~nm}$, respectively. For each $\lambda_{1}$, the MPEL is measured at two wavelength regions for a series of hole diameters. Dashed line indicated FWM for the same $\lambda_{1}$, showing clear similarities between FWM and MPEL in dependence on hole diameter. All results were acquired with the high hole density films.

around the nanoholes for better insights in the roles of the different surface plasmon modes.

The high FWM/MPEL ratio is promising for biosensing applications as MPEL otherwise could give a disturbing background in surface-enhanced CARS measurements. For such measurements the issues of separating the FWM generated by the gold film from the surfaceenhanced CARS signal and possible thermal damage of the sample from the film, needs to be resolved. For FWM generation in plasmonic structures with femtosecond coherence time, picosecond laser pulses are not optimal. Shorter laser pulses could enhance the nonlinear signals. Picosecond pulses have, however, a suitable bandwidth for vibrational sensitive CARS measurements [22], and could therefore be beneficial for future biosensing application.

In conclusion, we show large enhancements of nonlinear emission, including FWM, SHG, and broadband MPEL, by nanoholes in thin gold films excited by a conventional nonlinear microscope using high-NA laser scan excitation. Due to the small excitation volume, enhancement is restricted to when the holes are directly addressed, and even for short hole-hole distances the plasmons are excited through one hole at the time.
The conversion efficiency of the FWM shows a resonant behavior when the shorter (quadratic) pump/probe excitation wavelength and the HPR are matched. The locality of the nonlinear nanohole excitation opens for large scale production of high-density device arrays, where each hole can act as a separate nanosensor, emitter, frequency convertor, and/or antenna.

We gratefully acknowledge support from The Swedish Research Council, Chalmers Area of Advance Nanoscience and Nanotechnology, the Åforsk Foundation, the Wenner-Gren Foundations, and the Wallenberg Foundation.

\section{References}

1. A. D. McFarland and R. P. Van Duyne, Nano Lett. 3, 1057 (2003).

2. P. Muhlschlegel, H. J. Eisler, O. J. F. Martin, B. Hecht, and D. W. Pohl, Science 308, 1607 (2005).

3. S. I. Bozhevolnyi, V. S. Volkov, E. Devaux, and T. W. Ebbesen, Phys. Rev. Lett. 95, 046802 (2005).

4. H. Ditlbacher, A. Hohenau, D. Wagner, U. Kreibig, M. Rogers, F. Hofer, F. R. Aussenegg, and J. R. Krenn, Phys. Rev. Lett. 95, 257403 (2005).

5. A. B. Dahlin, S. Chen, M. P. Jonsson, L. Gunnarsson, M. Kall, and F. Hook, Anal. Chem. 81, 6572 (2009).

6. T. Sannomiya, O. Scholder, K. Jefimovs, C. Hafner, and A. B. Dahlin, Small 7, 1653 (2011).

7. R. A. Farrer, F. L. Butterfield, V. W. Chen, and J. T. Fourkas, Nano Lett. 5, 1139 (2005).

8. S. I. Bozhevolnyi, J. Beermann, and V. Coello, Phys. Rev. Lett. 90, 197403 (2003).

9. A. Lesuffleur, L. K. S. Kumar, and R. Gordon, Appl. Phys. Lett. 88, 261104 (2006).

10. M.Lippitz, M.A. van Dijk, and M. Orrit, NanoLett. 5, 799 (2005).

11. S. Kim, J. Jin, Y.-J. Kim, I.-Y. Park, Y. Kim, and S.-W. Kim, Nature 453, 757 (2008).

12. A. Bouhelier, M. Beversluis, A. Hartschuh, and L. Novotny, Phys. Rev. Lett. 90, 013903 (2003).

13. P. N. Melentiev, T. V. Konstantinova, A. E. Afanasiev, A. A. Kuzin, A. S. Baturin, and V. I. Balykin, Opt. Express 20, 19474 (2012).

14. X. Liu, Y. Wang, and E. O. Potma, Opt. Lett. 36, 2348 (2011).

15. J. Renger, R. Quidant, N. van Hulst, S. Palomba, and L. Novotny, Phys. Rev. Lett. 103, 266802 (2009).

16. S. Palomba and L. Novotny, Phys. Rev. Lett. 101, 056802 (2008)

17. H. Kim, D. K. Taggart, C. X. Xiang, R. M. Penner, and E. O. Potma, Nano Lett. 8, 2373 (2008).

18. C. Steuwe, C. F. Kaminski, J. J. Baumberg, and S. Mahajan, Nano Lett. 11, 5339 (2011).

19. P. Genevet, J.-P.Tetienne, E. Gatzogiannis, R. Blanchard, M.A. Kats, M. O. Scully, and F. Capasso, Nano Lett. 10, 4880 (2010).

20. M. P. Jonsson, A. B. Dahlin, P. Jonsson, and F. Hook, Biointerphases 3, Fd30 (2008).

21. T. Rindzevicius, Y. Alaverdyan, A. Dahlin, F. Hook, D. S. Sutherland, and M. Kall, Nano Lett. 5, 2335 (2005).

22. J.-X. Cheng and X. S. Xie, Coherent Raman Scattering Microscopy (CRC Press, 2013).

23. P. Hanarp, D. S. Sutherland, J. Gold, and B. Kasemo, Colloids Surf. A 214, 23 (2003).

24. A. Enejder, C. Brackmann, and F. Svedberg, IEEE J. Sel. Top. Quantum Electron. 16, 506 (2010).

25. J. Alegret, P. Johansson, and M. Kall, New J. Phys. 10, 105004 (2008).

26. T. H. Park, N. Mirin, J. B. Lassiter, C. L. Nehl, N. J. Halas, and P. Nordlander, ACS Nano 2, 25 (2008). 